\title{
Effects of Number of Nodes and Network Area Size Parameters on WSN Protocols Performances
}

\author{
Hamdy H. El-Sayed \\ Faculty of Computers and Information, Sohag University, Egypt. \\ hamdy2006x@gmail.com
}

ABSTRACT

Wireless sensor network (WSN) consists of small devices, which are called sensors. It is capable of sensing the environmental events, make processing of them and send data to the base station (BS), which needs high energy for its usage. This network which is limited to iterate the dead nodes, bring by energy depletion and to maximize the life-span of the system. Many routing protocols have been proposed and the efficiency of WSN declines as changing of the parameters of sensor nodes. The protocols in WSN are classified to heterogeneous or homogeneous. In this paper, we test the effects of node density and network area on various distributed energyefficient clustering based on protocols such as Distributed Energy-Efficient Clustering (DEEC), Developed Distributed Energy-Efficient Clustering (DDEEC) and Threshold Distributed Energy-Efficient Clustering (TDEEC) as multilevel heterogeneous protocols, and MODLEACH protocols as an example of homogeneous routing protocols. Threshold Distributed Energy-Efficient Clustering protocol has better performance than Distributed Energy-Efficient Clustering protocol, Distributed Energy-Efficient Clustering protocol and Enhanced Distributed Energy-Efficient Clustering protocol (EDEEC) but Modified Low Energy Adaptive Clustering Hierarchy protocol (MODLEACH) is lengthy the stable period other than protocols. The sent packet to BS and the received one from BS are increased with increasing of nodes number and decreased with increasing of network area. The life time of network decreases conversely with increasing the area of transmission. These parameters will increase the performance of the entire network. Especially in real-time applications that use the WSNs, which, are expected to work in fields such as industry, rubout or battle tracking.

Keywords - Energy-Efficient Clustering Protocol, Wireless Sensor Networks, Heterogeneous and homogeneous protocols, Distributed algorithms, Network performances.

\section{INTRODUCTION}

In contemporary wireless sensor network (WSN) applications, the entire network must be capable of operating unattended in ruthless environments in which pure human login and control neither be easily scheduled efficiently managed nor it is even feasible at all [1]. From this critical situation, our expectation, -in all of significant WSN applications- the sensor nodes are often deployed randomly in the area that we are interested in relatively free. Also, they conform a network in an ad hoc manner $[2,3]$. They suffer from various factors such as low bandwidth, high error rate, more frequent link breakage, end-to-end delay, and high loss of pocket, etc. Furthermore, considering the entire area that has to be covered, the short life of the battery energy of the sensors, the probability of damaged nodes during deployment and large amount of sensors are expected; it is a normal probability that hundreds or even thousands of sensor nodes will be involved. Thus, sensors in such environments are energy-constrained and usually their batteries couldn't be recharged. Therefore, it is clearly that the special energy-aware routing and data gathering protocols offer high scalability. These observations are recommended to be applied in order to conserve that network lifetime in such environments.
Naturally, group of sensor nodes into clusters have been exceedingly adopted by the research community to be involved with the above scalability objective and generally realized high energy efficiency and expanded network lifetime in huge WSN environments. The conformable hierarchical routing and data gathering protocols hint cluster-based organization of the sensor nodes regular that data fusion and aggregation are possible, thus lead to save energy. In the hierarchical network structure, each cluster has a head, which is also called the cluster head $(\mathrm{CH})$ and usually performs the aforementioned special tasks (fusion and aggregation), and several common sensor nodes (SN) as members.

The BS refers to the data processing point that is received from the sensor nodes, where the data is accessed by the final user. Mainly, it is fixed at far distance from the sensor nodes. The $\mathrm{CH}$ nodes worked as gateways between the sensor nodes and the BS. The function of each $\mathrm{CH}$, which mentioned above, is to perform common functions for all the nodes in the cluster, such as aggregating the data before sending it to the BS. Anyway, the $\mathrm{CH}$ is the sink for the cluster nodes, and the BS is the sink for the CHs. Clustering could be performed in two types of networks, which are homogenous and heterogeneous. Nodes which have the same energy level are called homogenous network and those which have different energy levels are called heterogeneous ones. Various 
algorithms for homogenous network in WSN are LowEnergy Adaptive Clustering Hierarchy (LEACH), Power Efficient Gathering in Sensor Information Systems (PEGASIS) [4], and Hybrid Energy-Efficient Distributed clustering (HEED) [5]. On the other hand, another algorithms like Stable Election Protocol (SEP) [6], Distributed Energy-Efficient Clustering (DEEC) [7], Developed DEEC (DDEEC) [8], Enhanced DEEC (EDEEC) [9], Threshold DEEC (TDEEC)[10] and Threshold Sensitive Advanced DEEC (TADEEC)[11] are for heterogeneous protocols of WSNs. SEP is designed for two level heterogeneous networks, so it does not worked efficiently in three or multilevel heterogeneous network. DEEC, DDEEC, EDEEC, TDEEC and TADEEC are contagious for multilevel heterogeneous networks and can also perform efficiently in two level heterogeneous scenarios.

The MODLEACH "Modified Low Energy Adaptive Clustering Hierarchy" which is a variant of LEACH has the efficient head replacement scheme and dual transmission power level which will help in the energy conservation of the wireless sensor network [12].

In this paper, performance of various clustering protocols under three and multilevel heterogeneous networks is discussed. Nodes number and network area are two parameters that have been used to test the performance of DEEC, DDEEC, EDEEC and TDEEC for different scenarios of three and multilevel heterogeneous WSNs. Three level heterogeneous networks contain normal, advanced and super nodes, whereas the later has highest energy level comparing with normal and advanced nodes. A MODLEACH protocol is an example of homogeneous networks which have been compared with Distributed Energy Efficient Clustering Protocols. These protocols have been compared under the similar environments with different number of nodes and different network area of transmission. We observed that the TDEEC protocol performs better than other Distributed Energy Efficient Clustering Protocols and the MODLEACH protocol is more stable.

In section 2 of this paper we discuss an overview of Distributed Clustered Protocols of WSN. In Section 3. The define of MODLEACH protocol is presented. Section 4. Consists of simulation and discussion and in section 5 Contribution and Conclusion are presented. Lastly in Section 6 References are presented.

\section{2 - Overview of Distributed Clustered Protocols of WSN}

Heterogeneous WSN consists of sensor nodes having different energy levels. This kind of sensor network contains two, three or multi types of sensor nodes with respect to their energy levels(Two level heterogeneous WSN model or Three level Heterogeneous WSN). Pankaj Kumar and Dr. N. C. Barwar [13] studied these models and introduced a mathematical background for DEEC,
DDEEC, EDEEC and TDEEC protocols. We compare the differences between these protocols as follow.

\section{1- DEEC}

In DEEC, the cluster-heads are elected by a probability due to the ratio between residual energy of each node and the average energy of the network. The epochs of being cluster-heads for nodes are different according to their initial and residual energy. The nodes with high initial and residual energy have chances to be the clusterheads more than the nodes with low energy [7].

\section{2- DDEEC}

DDEEC uses the same method for estimation of average energy in the network and $\mathrm{CH}$ selection algorithm is based on residual energy as implemented in DEEC [8].

\section{3-EDEEC}

EDEEC uses a concept of three level heterogeneous networks. It contains three types of nodes which are normal, advanced and super nodes due to initial energy. It is evaluated [14] that EDEEC performs better than DDEEC[9].

\section{4-TDEEC}

TDEEC uses the same mechanism for $\mathrm{CH}$ selection and average energy estimation, which are implemented in DEEC. At each round, nodes decide whether to become a $\mathrm{CH}$ or not by choosing a random number between 0 and 1 . If number is less than

threshold Ts which is shown below, then node becomes $\mathrm{CH}$ for the given round. In TDEEC, threshold value is adjusted based on that value; a node decides whether to become a $\mathrm{CH}$ or not by introducing residual energy and average energy of that round regarding the optimum number of CHs. Threshold value is proposed in [15].

\section{3- MODLEACH Protocols}

The energy consumption will affect properly the performance of the WSN as it does not define the life time of the node only, but also affects the frequency of the cluster formation and spent resources on the cluster head formation[16]. The LEACH protocol stands for " Low Energy Adaptive Clustering Hierarchy" [17] is TDMA based on MAC protocol with the idea of low energy consumption to maintain cluster and to extend the life of the WSN. The MODLEACH is "Modified Low Energy Adaptive Clustering Hierarchy"[18,19] which is a variant of LEACH has the efficient head replacement scheme and dual transmission power level which will help in the energy conservation of the wireless sensor network.

\section{4- Simulation and Discussion}

We simulate different Distributed Energy Clustering protocols in heterogeneous and homogeneous WSNs using MATLAB. For simulation, we use the parameters in the previous table and various number of nodes 
$500,1000,2000$ and different network area $150 * 150$, $200 * 200,300 * 300$. We considered the BS placed the center of the network field as shown in Table 1.

The studied effects of changing parameters used for evaluation of clustering protocols for heterogeneous WSNs are lifetime of heterogeneous WSNs, number of nodes alive during rounds and data packets sent to BS.

1- Dead nodes: are nodes which get died until last round, thus, better network performance will be with minimum dead nodes.

2- Alive nodes: are nodes which exist until the last round. Accordingly, the higher alive nodes the better obtained performance.

3- Data packets sent to the BS: indicate quantity of packets received by BS for each round.

4. Cluster Head $\mathrm{CH}$ packets: indicate the quantity of sent packets to BS for each round.

Table 1: list of simulation parameters

\begin{tabular}{|c|c|c|}
\hline $\begin{array}{c}\text { S. } \\
\text { No. }\end{array}$ & Parameters & Values \\
\hline 1 & Network Area & $100 * 100,200 * 200,300 * 300$ \\
\hline 2 & Number of nodes & $500,1000,2000$ \\
\hline 3 & Cluster head probability & 0.1 \\
\hline 4 & Base station location & $(\mathbf{5 0 , 5 0 )}$ \\
\hline 5 & Transmiter energy & $50 * 0.000000001$ \\
\hline 6 & Reciever energy & $50 * 0.000000001$ \\
\hline 7 & Aggregation energy & $5 * 0.000000001$ \\
\hline 8 & Amplification energy & $0.0013 * 0.000000000001$ \\
\hline 9 & Number of rounds & 3000 \\
\hline 10 & Hard threshold & 100 \\
\hline 11 & Soft threshold & 2 \\
\hline
\end{tabular}

\section{A-Number of node changes}

1- Run of the network simulation with 500 nodes, network area $100 * 100$ and number of round 3000 round on DEEC, Developed DEEC, Enhanced DEEC, Threshold DEEC and MODLEACH protocols.
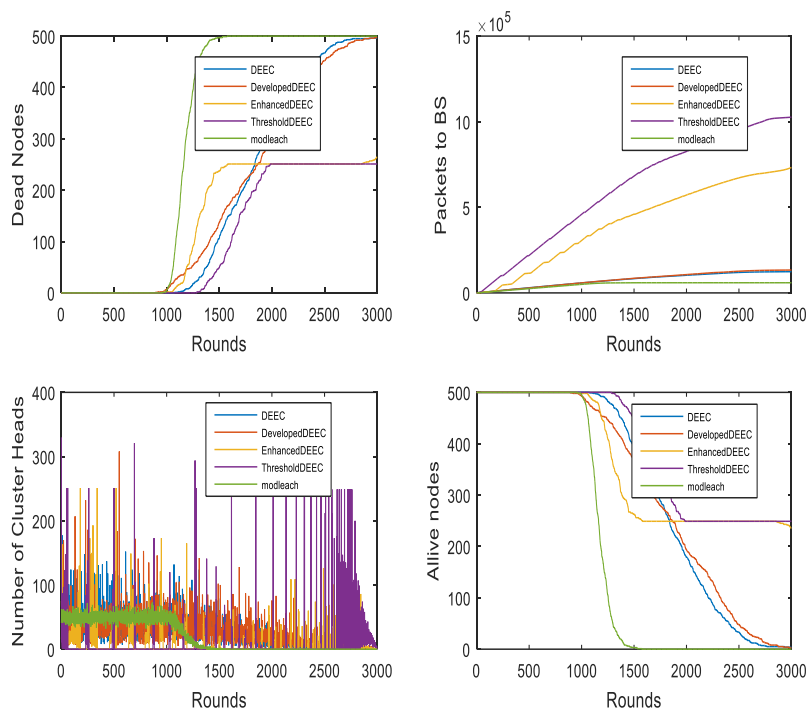

Figure 1: Dead Nodes, Alive Nodes, Packet sends to BS Nodes, Count of Cluster Head per round during 3000 rounds and 500 nodes.
2- Run of the network simulation with 1000 nodes, network area $100 * 100$ and number of round 3000 round on DEEC, DDEEC, EDEEC, TDEEC and MODLEACH protocols.
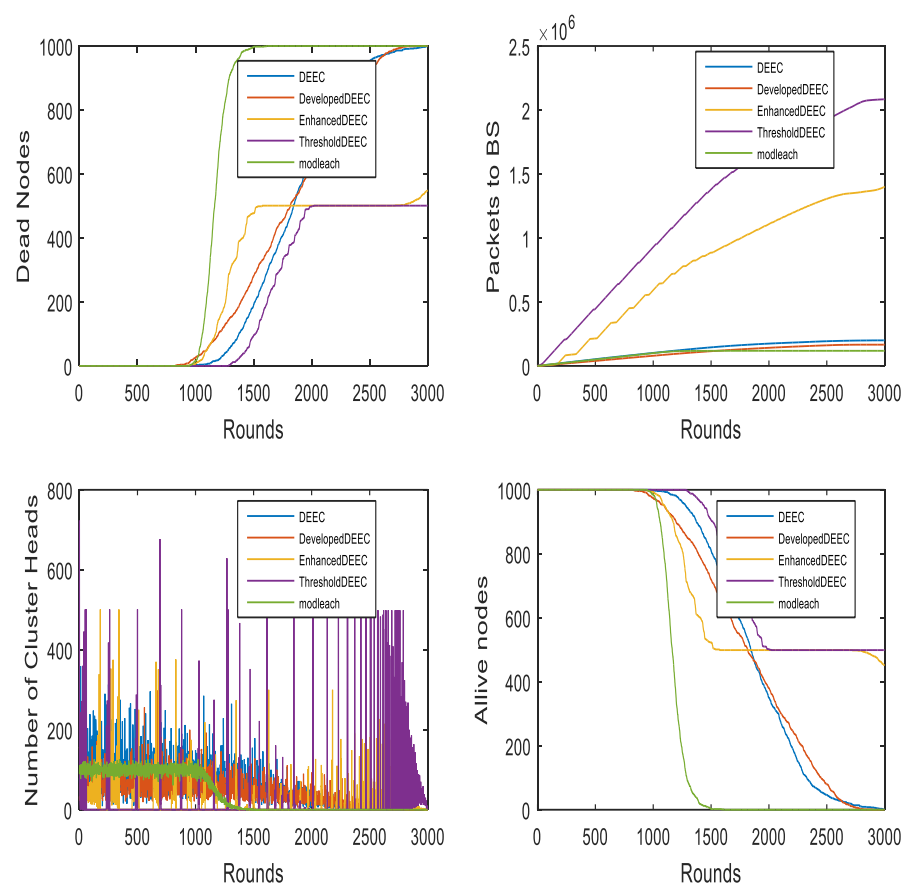

Figure 2: Dead Nodes, Alive Nodes, Packet sends to BS Nodes, Count of Cluster Head per round during 3000 rounds and 1000 nodes.

3- Run of the network simulation with 2000 nodes, network area $100 * 100$,number of round 3000 round on DEEC, DDEEC, EDEEC, TDEEC and MODLEACH protocols.
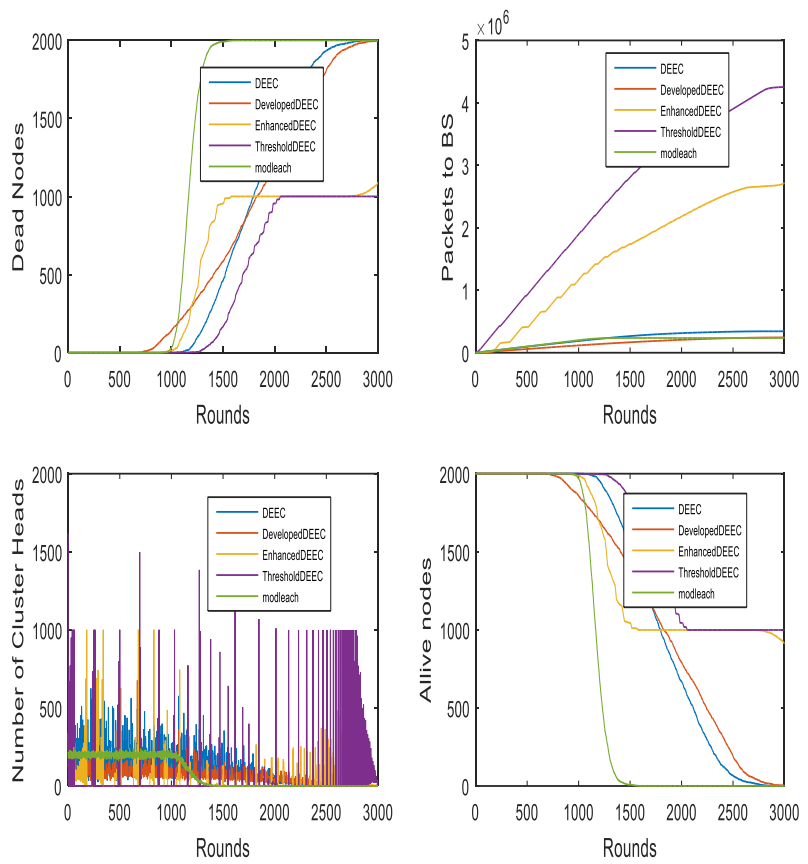

Figure 3: Dead Nodes, Alive Nodes, Packet sends to BS Nodes, Count of Cluster Head per round during 3000 rounds and 2000 nodes. 


\section{B-Area of network changes.}

1- Run of the network simulation with 1000 nodes, network area $150 * 150$ and number of round 3000 on DEEC protocols.
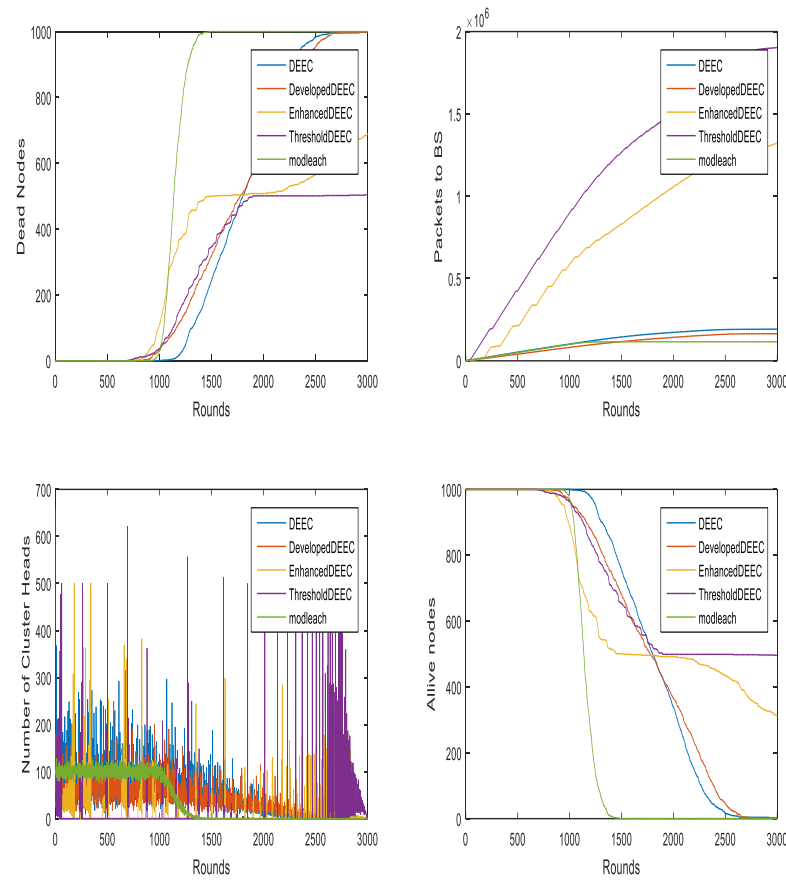

Figure 4: Dead Nodes, Alive Nodes, Packet sends to BS Nodes, Cluster Head per round during 3000 rounds and 1000 nodes with $150 * 150$ network area.

2- Run of the network simulation with 1000 nodes, network area $200 * 200$ and number of round 3000 on DDEEC protocols.
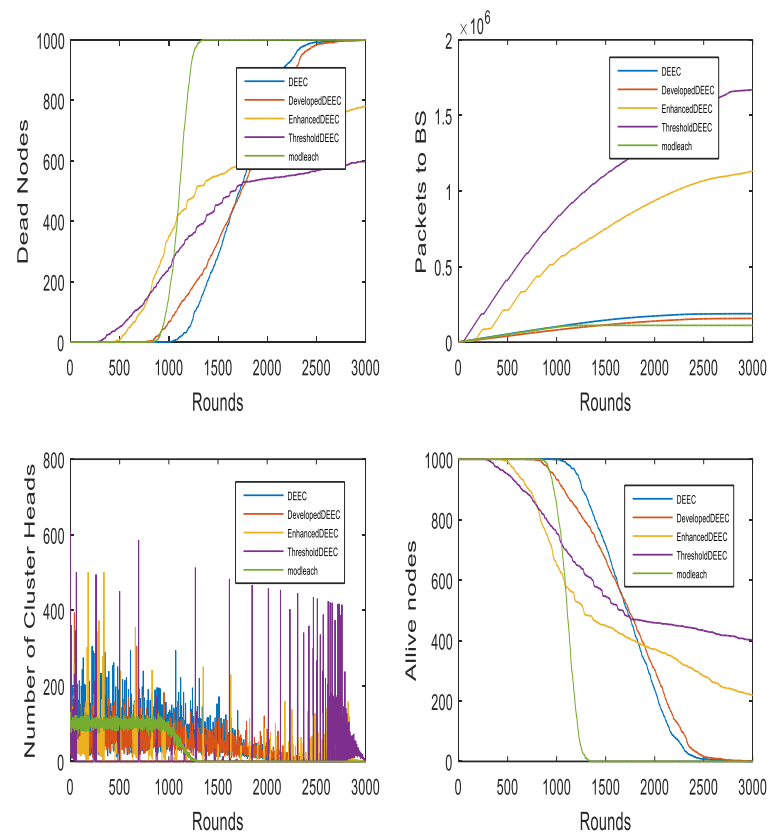

Figure 5: Dead Nodes, Alive Nodes, Packet sends to BS Nodes, Count of Cluster Head per round during 3000 rounds and 1000 nodes with 200* 200 network area.
3- Run of the simulation network with 1000 nodes, network area $300 * 300$ and number of round 3000 on EDEEC protocols.
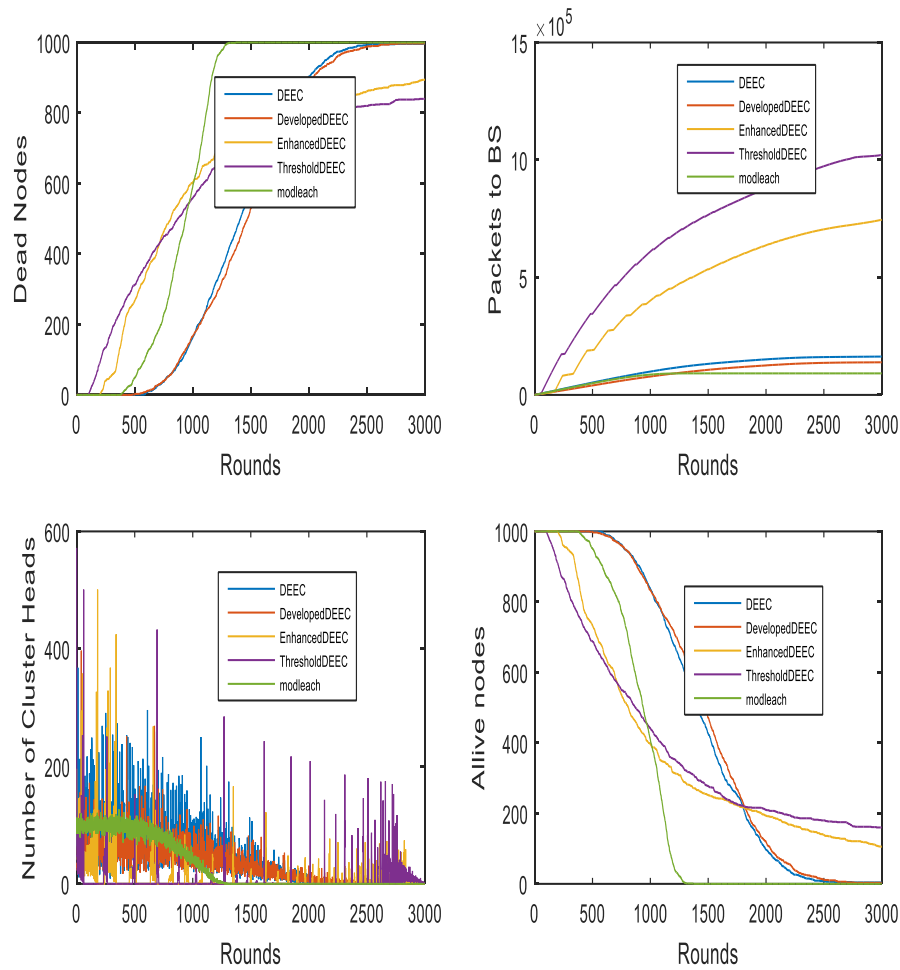

Figure 6: Dead Nodes, Alive Nodes, Packet sends to BS Nodes, Count of Cluster Head per round during 3000 rounds and 1000 nodes with 300*300 network area.

\section{1 - Discussion \\ A- Number of Node Changes.}

The simulation run to examine the DEEC protocols with different number of nodes 500,1000 and 2000 in the same network area as observed from figure 1, that the first_dead nodes dies at 1094, the tenth_dead dies at 1329, and all_dead nodes dies at 16840 ,rounds respectively. The packet to send to $\mathrm{CH}$ is 796607 also the packet to send to BS is 125187. Figure 2 shows that first_dead nodes dies at 1079, tenth_dead dies at 1386 , and all_dead dies at 16930, rounds respectively. The packet that is sends to $\mathrm{CH}$ is 802671 also the packet send to BS is 124639. Figure 3 depicts that first_dead nodes dies at 1027, tenth_dead dies at 1349, and all_dead dies at 16910 , rounds respectively. The packet to $\mathrm{CH}$ is 3331347; also the packet send to BS is 343862. The first dead nodes decreased inversely with increased number of nodes while the tenth dead nodes and all dead nodes increased with increasing number of nodes. The sent packet to BS and the received packet are of BS having been increased with increasing number of nodes.

Similarly, the simulation run to examine the DDEEC protocols with different number of nodes 500,1000 and 2000 in the same network area as observed from figure 1 that the first_dead nodes dies at 900, the tenth_dead dies at 1212, and all_dead dies at 1540, rounds 
respectively. The packet sends to $\mathrm{CH}$ is 792694 , and the packets that are send to BS are 128262. Figure 2 shows that first_dead nodes dies at 825, the tenth_dead dies at 1162, and all_dead nodes dies at 1430, rounds respectively. The packets are send to $\mathrm{CH}$ are 1668201 , also the packet sends to BS are 172004. Figure 3, depicts that the first_dead nodes dies at 593, the tenth_dead dies at 1128, and all_dead nodes dies at 1490, rounds respectively. The packet that are sends to $\mathrm{CH}$ are 3434087 , also the packet sends to BS are 23561. the first dead nodes decreased with increased number of nodes also the teenth dead nodes and all dead nodes decreased with increasing number of nodes. The sent packet to BS and the received packet of BS have been increased with increasing number of nodes.

The EDEEC protocols are simulated with various number of nodes 500,1000 and 2000 in the same network area as observed from figure 1 that the first_dead nodes dies at 1031, the tenth_dead dies at 1205, and all_dead nodes dies at 1530, rounds respectively. The packets that are sends to $\mathrm{CH}$ are 339400 , also the packet that are sends to BS are 734497. Figure 2 also depicts that first_dead nodes dies at 745, the teenth_dead dies at 1174, and the all_dead nodes dies at 1488 , rounds respectively. The packets that are sends to $\mathrm{CH}$ are 1542489 , also the packet that are sends to BS are 271743. Figure 3 depicts that the first_dead nodes dies at 850 , the teenth_dead dies at 1179 , and all_dead nodes dies at 1510 , rounds respectively. The packet that are sends to the $\mathrm{CH}$ are 739186 , also packets that are sends to BS are 1396863. The first dead nodes decreased with the increasing number of nodes. Also, the tenth dead nodes and all dead nodes decreased with the increasing number of nodes. The sent packet to BS and the received packet of $\mathrm{BS}$ are increased with increasing number of nodes.

In the TDEEC protocols, which are simulated and examine with different number of nodes 500,1000 and 2000 in the same network area, that was observed from figure 1 that first_dead dies at 1239, tenth_dead dies at 1508, and all_dead dies at 1760, rounds respectively. The packts that are sends to $\mathrm{CH}$ are 134945 , also the packets that are sends to BS are 1029189. Figure 2 revealed the first_dead nodes at 1263, tenth_dead nodes dies 1530 , and all_dead dies at 1806, rounds respectively. The packet that are sends to packet to $\mathrm{CH}$ are 246886, also the packet sends to BS are 2090399. Figure 3 depicts that first_dead dies at 1155, teenth_dead dies at 1544, and all_dead dies at 1844 , the packet are sends to $\mathrm{CH}$ are 430356, the packet that are sends to BS are 4276053. The first dead nodes increased with number of nodes 1000 and decreased with the increasing number of nodes to 2000 also the tenth dead nodes and all dead nodes decreased with increasing number of nodes. The sent packet to BS and the received packet of BS are increased with increasing number of nodes.
The simulation that runs to examine the MODLEACH protocol as an example of homogenous protocols with different number of nodes 500, 1000. And 2000 in the same network area as observed from Figure 1, that the first_dead dies at 1014, the tenth_dead dies at 1110, and all_dead dies at 1561 rounds respectively. The packets that are sends to $\mathrm{CH}$ are 553582 and the packet sends to BS are 62053. Figure 2, depicts that the first_dead dies at 1012, the tenth_dead dies at 1113, and the all_dead dies at 1615 , rounds respectively. The packet that are sends to $\mathrm{CH}$ are 1107799, and also the packet that has been sends to BS are 123740. Figure 3, shows that the first_dead dies at 971 , the tenth_dead dies at 1122, and all_dead dies at 1658 , rounds respectively. The packet that has been send to $\mathrm{CH}$ is 2216055 and packets that send to BS are 247034 . The first_dead nodes slightly decreased with increasing number of nodes. Similarly, the tenth dead nodes and all dead nodes slightly decreased with increasing number of nodes. The sent packet to BS and the received packet of $\mathrm{BS}$ are increased with increasing number of nodes.

From the previous simulations, we observed that lifetime of network nodes increased with increasing number of nodes in the network. In addition, the round number when the first node dies is almost the same in all cases, but the total number of nodes which die after the completion of simulations increases with the number of nodes and similarly with the case of number of temporal cluster heads, packet sent to BS and alive nodes.

The cluster formation of the MODLEACH shows that the MODLEACH has the cluster formation stability lower than DEEC, DEEC,EDEEC and TDEEC protocols. TDEEC has better performance than DEEC, DEEC and EDEEC. However, MODLEACH is lengthy the stable period (which means that the period of time before first node dead). DEEC and DDEEC have high effect by changing number of nodes in the network. The packet transmission rate of MODLEACH is less than Distributed Energy Efficient Clustering Protocols. However, life time is extended in MODLEACH. EDEEC protocols performance better than DDEEC in several of network nodes. The rate of packet transmission of homogeneous WSN (MODLEACH) is less than other multilevel heterogeneous WSN (DEEC, DDEEC, EDEEC and TDEEC). The energy efficiency of the MODLEACH protocol is less than other protocols, while the network life extended in MODLEACH.

\section{B-Network Area Changes}

The simulation in this section runs with run number of Nodes 1000 and network area changes in square dimensions like $150 * 150,200 * 200$ and $300 * 300$.

The simulation runs to examine the DEEC protocols as observed from figure 4, that the first_dead dies at 1006 , the tenth_dead dies at 1317, and all_dead dies at 1692, rounds respectively. The packet has been send to $\mathrm{CH}$ are 
1607251 and the packet that are send to BS are 194380. Figure 5, depicts that the first_dead dies at 949, the tenth_dead dies at 1271, and all_dead dies at 1598, rounds respectively. The packet that has been send to $\mathrm{CH}$ are 1529561 and the packet sends to BS are 184067. Figure 6 revealed that the first_dead dies at 453, teenth_dead dies at 887, and all_dead dies at 999, rounds respectively. The packet that are sends to $\mathrm{CH}$ are 1232312 and the packet are sends to BS are 156864 .

The first dead nodes decreased with the increasing of network area. Also, the tenth dead nodes and all dead nodes decreased with the increasing network area. The sent packet to BS and the received packet of BS are decreased with increasing network area. The life time of network decreases with increasing the network area.

Similarly as the examination of DDEEC protocol it was observed from figure 4, that the first_dead dies at 783, the tenth_dead dies at 1143 , and all_dead dies at 1501, rounds respectively. The packet which has been sent to $\mathrm{CH}$ is 1630803 and the packet that has been sends to BS are 168288. Figure 5, depicts that the first_dead dies at 757, the tenth_dead dies at 1093, and all_dead dies at 1260 , rounds respectively. The packet that has been received by $\mathrm{CH}$ are 1551752 and the packet where sends to the BS are 164381. Figure 6, shows that the first_dead dies at 473, the tenth_dead dies at 888, and all_dead dies at 995 . The packet which received by $\mathrm{CH}$ are 1335906 and the packet sends to the BS are 140592. The first dead nodes decreased with increased network area. Also, the tenth dead nodes and all dead nodes decreased with increasing network area. The sent packet to BS and the received packet of $\mathrm{BS}$ are decreased with increasing network area. The life time decreases with increasing the network area. It is observed that the changes of network area affect the performance.

On the other hand, from the simulation that has been run to examine the EDEEC protocol, we observed from figure 4 that the first_dead dies at 751, the tenth_dead dies at 973, and all_dead dies at 1170, rounds respectively. The packet which received by $\mathrm{CH}$ are 648563, and the packet which send to BS are 1321351. Figure 5, shows also that the first_dead dies at 430, the tenth_dead dies at 717, and all_dead dies at 780, rounds respectively. The packet which are sends to $\mathrm{CH}$ are 556466 and the packet that has been sends to BS are 1139003. Figure 6, depicts also the first_dead dies at 195, the tenth_dead dies at 360 , and all_dead dies at 780 , rounds respectively. The packets that are sending to $\mathrm{CH}$ are 408573 and the packet that has been send to the BS is 741948 .

The first dead nodes decreased with increased network area. Additionally, the tenth dead nodes and all dead nodes decreased with increasing network area. The sent packet to BS and the received packet of BS are decreased with increasing network area. The life time decreases with increasing the network area. The different network area effects on the performance for different parameters.

Simulation that has been examine the TDEEC protocol performance shows in figure $\mathbf{4}$, the first_dead dies at 671, the tenth_dead dies at 1093, and all_dead dies at 1320 , rounds respectively. The packet which received by $\mathrm{CH}$ are 264311 and the packet sends to BS are 1894708 . Figure 5 shows the first_dead dies at 318, the tenth_dead dies at 657, and all_dead dies at 807, rounds respectively. The packet which received by $\mathrm{CH}$ are 243225 and the packet that has been send to BS are 1664774. Figure 6 reveals that the first_dead dies at 102 , the tenth_dead dies at 223, and all_dead dies at 838, rounds respectively. The packets which are received by $\mathrm{CH}$ are 162913 and the packet that are sends to BS are 1033394.

The first dead nodes decreased with the increasing network area. Also, the tenth dead nodes and all dead nodes decreased with increasing network area. The packet sends to BS and the received packets of BS are decreased with increasing network area. The life time decreases with increasing the network area. The different network area effects on the performance for different parameters.

Finally, from the simulation that has been run to examine the MODLEACH protocol we observed from figure 4 that the first_dead dies at 957, tenth_dead dies at 1095, and all_dead dies at 1522, rounds respectively. The packets that have been sending to $\mathrm{CH}$ are 1085531 and the packets are sends to BS are 121315. Figure 5, shows that the first_dead nodes die ate 824, the tenth_dead dies at 1004, and all_dead dies at 1539, rounds respectively. The packet sends to $\mathrm{CH}$ are 1034505 and the packet that has been send to BS is 115553 . Figure 6, depicts that the first_dead nodes dies at 407, the tenth_dead dies at 612 , and all_dead dies at 1502 , rounds respectively. The packets that have been sends to $\mathrm{CH}$ are 850552 and packets that are sends to BS are 95341.

The first dead nodes decreased with the increasing network area. Also, the tenth dead nodes and all dead nodes decreased with the increasing network area. The sent packet to BS and the received packet of BS are decreased with the increasing network area. The life time decreases with increasing the network area. The different network area effects on the performance for different parameters.

The simulation with network area changes the effects on the performance of DEEC, DDEEC, EDEEC, TDEEC and MODLEACH protocols. Based on the obtained results, we conclude that all dead nodes decreased with increasing network area. Similarly sent packet and received one to BS are decreased with increasing network area. The life time decreased with increasing area of transmission. We also conclude that EDEEC and TDEEC have better performance among the Distributed Energy Clustering Protocols in WSN. However, MODLEACH protocol 
as an example of homogenous WSN is more stable other than different Distributed Energy Clustering Protocols.

\section{5- Contribution and Conclusion}

This paper examined the different distributed energy clustering protocols such as DEEC, DDEEC, EDEEC and TDEEC and MODLEAC, which have been modified from LEACH protocols and considered as an example of homogeneous WSN protocols.

It is observed according to changing number of nodes in the network, the first dead, tenth dead and all dead are decreased with increasing number of nodes, the packet send to $\mathrm{BS}$ and received packet are increasing with increasing number of nodes. In the network area changes, the first dead, tenth dead and all dead are decreased a lot with increasing area of network as well as the sent packet to $\mathrm{BS}$ and received packet are decreased too. The life time of network decreased with increasing network area. The number of tentative cluster head formed in the network for any simulation increased with increasing number of nodes. We conclude that both changing number of nodes and area of network have clear effects on the performance of heterogeneous protocols and homogeneous protocols. Moreover, these parameters will increase the performance of the network quality. It will be affected in all applications that use the WSNs. It is expected to work in fields such as industry, rubout or battle tracking. The modeling of these protocols seems to suggest that the node density and network area have clear effects on the nodes in cluster head and must be taken into consideration during the process design. In future of design and process of the classification of these protocol must take in consideration these parameters because these protocols will be efficient for applications that are time critical by nature.

\section{REFERENCES}

[1] A.A. Abbasi and M. Younis, "A survey on clustering algorithms for wireless sensor networks", Computer Communications, 30, 2007, 2826-2841.

[2] K. Sohrabi et al., "Protocols for self-organization of a wireless sensor network", IEEE Personal Communications, 2000,7(5), 16-27.

[3] R. Min et al., "Low power wireless sensor networks", in Proceedings of International Conference on VLSI Design, Bangalore, India, January 2001.

[4] S. Lindsey, C.S. Raghavenda, "PEGASIS: power efficient gathering in sensor information systems", in: Proceeding of the IEEE Aerospace Conference, Big Sky, Montana, March 2002.

[5] O. Younis, S. Fahmy, HEED: "A hybrid, energyefficient, distributed clustering approach for ad hoc sensor networks", IEEE Transactions on Mobile Computing 3 (4) , 2004, 660-669.
[6] G. Smaragdakis, I. Matta, A. Bestavros, "SEP: A Stable Election Protocol for clustered heterogeneous wireless sensor network", in: Second International Workshop on Sensor and Actor Network Protocols and Applications (SANPA 2004), 2004.

[7] L. Qing, Q. Zhu, M. Wang, "Design of a distributed energy efficient clustering algorithm for heterogeneous wireless sensor network", ELSEVIER, Computer Communications 29, 2006, pp 2230- 2237.

[8] Elbhiri B., Saadane R., El Fkihi,S., Aboutajdine, D. "Developed Distributed Energy-Efficient Clustering (DDEEC) for heterogeneous wireless sensor networks", in: 5th International Symposium on I/V Communications and Mobile Network (ISVC), 2010.

[9] Parul Saini, Ajay.K.Sharma, "E-DEEC- Enhanced Distributed Energy Efficient Clustering Scheme for heterogeneous WSN", in: 2010 1st International Conference on Parallel, Distributed and Grid Computing (PDGC) 2010.

[10] Parul Saini, Ajay. K. Sharma, "Energy Efficient Scheme for Clustering Protocol Prolonging the Lifetime of Heterogeneous Wireless Sensor Networks", International Journal of Computer Applications (09758887), September 2010.

[11] Anamika Chauhan and Amit Kaushik, "TADEEC: Threshold Sensitive Advanced Distributed Energy Efficient Clustering Routing Protocol for Wireless Sensor Networks," International Journal of Computer Applications , Volume 96 No.23 June 2014, 0975 8887.

[12] D. Mahmood, N. Javaid, S. Mahmood, S. Qureshi, A. M. Memon, and T. Zaman, "MODLEACH: A variant of LEACH for WSNs", in Proc. $8^{\text {th }}$ Int. Conf. Broadband Wireless Comput., Commun. App.(BWCCA), Oct.2013, pp. 158-163,

[13] Pankaj Kumar and Dr. N. C. Barwar, "Performance Evaluation of Distributed Energy Efficient Clustering Protocols in Wireless Sensor Networks", International Journal on Recent and Innovation Trends in Computing and Communication Volume: 3 Issue: 12 December 2015.

[14] T. N. Qureshi, N. Javaid, M. Malik, U. Qasim, Z. A. Khan, "On Performance Evaluation of Variants of DEEC in WSNs", In Seventh International Conference on Broadband, Wireless Computing, Communication and Applications 2012.

[15] Rajeev Kumar, Rajdeep Kaur "Evaluating the Performance of DEEC variants", International Journal of Computer Applications Volume 97-No. 7, July 2014, 0975-8887. 
[16] Debabrata Singh and Sanjeet Kumar Nayak, "Enhanced modified LEACH (EMODLEACH) protocol for WSN", conference of Advanced Computing and Communication (ISACC), 2015 International Symposium on, IEEE, Sept. 2015 , Page(s): 328 - 333, 14-15.

[17] W. Heinzelman, A. Chandrakasan, and $\mathrm{H}$. Balakrishnan,"Energy-EfficientCommunication Protocols for Wireless Microsensor Networks". In Proceedings of Hawaiian International Conference on Systems Science, January 2000.

[18] Tenzin Jinpa and Dr. B.V.R Reddy, "The Study of the Energy Efficient Protocols (MODLEACH, SEP and DEEC)", International Journal of Computer Science \& Communication Networks, Vol 5(1), 2015, 32-38.

[19] Hamdy.H El-Sayed : " Data Aggregation Energy and Probability Effects on the Performance of EDEEC and MODLEACH Protocol in WSN", Applied Mathematics \& Information Sciences An International, Appl. Math. Inf. Sci. 12, (2018), No. 1, 1-7.

\section{Biographies and Photographs}

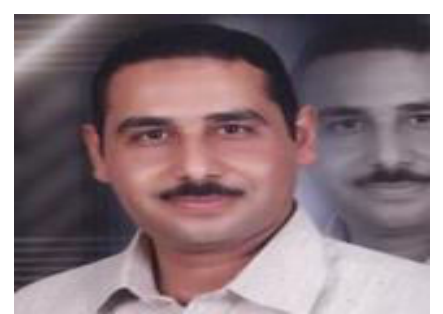

Hamdy H. El-Sayed received the PhD degree in wireless ad hoc network routing protocols from computer science department sohag university egypt march ,2015 . His research interests are in the areas of ad hoc routing protocols and sensor networks, cloud computing and security. 